\section{Apgar quirúrgico como criterio de activación de código 77 en el Hospital Ángeles Pedregal}

\author{
Surgical Apgar score as a criteria for activation of \\ code 77 at Angeles Pedregal Hospital
}

\author{
Dra. Erika Ramírez-Patiño, * Dr. José Jesús Alarcón-Rodríguez, ${ }^{\ddagger}$ \\ Dra. Jeanette Fabiola Schlitt-Niño, ${ }^{\S}$ Dr. Pedro Alejandro Elguea-Echavarría, \\ Dr. José Manuel Portela-Ortiz"
}

Citar como: Ramírez-Patiño E, Alarcón-Rodríguez JJ, Schlitt-Niño JF, Elguea-Echavarría PA, Portela-Ortiz JM. Apgar quirúrgico como criterio de activación de código 77 en el Hospital Ángeles Pedregal. Rev Mex Anestesiol. 2022; 45 (2): 82-86. https://dx.doi.org/10.35366/103881

\begin{abstract}
RESUMEN. Introducción: El Apgar quirúrgico (AQ) es un sistema de 10 puntos que valora tres variables intraoperatorias: frecuencia cardíaca más baja, presión arterial media más baja y sangrado. El AQ es un indicador de la mortalidad y morbilidad en el postoperatorio inmediato. Objetivo: Describir la incidencia del puntaje AQ en pacientes postoperados y con activación del código 77 en el Hospital Ángeles Pedregal. Material y métodos: Estudio observacional, descriptivo, retrospectivo, de corte transversal que incluyó pacientes con indicación de activación del código 77 en su postoperatorio inmediato y traslado a Unidad de Cuidados Intensivos, durante el período de enero a diciembre de 2015 en el Hospital Ángeles Pedregal. Resultados: Se contó con 58 pacientes de los cuales 68\% obtuvieron un Apgar quirúrgico $\geq 7$ puntos (estables) y $32 \%$ un Apgar quirúrgico 6 (inestables). La incidencia del Apgar quirúrgico 6 para el período de estudio fue de 12 casos por cada 100 personas intervenidas con una sensibilidad del $68 \%$. Conclusiones: EI $A Q$ puede considerarse un criterio confiable para la activación del código 77 en aquellos pacientes con puntaje $A Q$ $\leq 6$ durante el postoperatorio inmediato, permitiendo un manejo oportuno de las complicaciones postquirúrgicas y la disminución de riesgo de morbimortalidad.
\end{abstract}

ABSTRACT. Introduction: The surgical Apgar score (SAS) is a 10-point system that assesses three intraoperative variables: lower heart rate, lower mean arterial pressure, and bleeding. SAS is an indicator of mortality and morbidity in the immediate postoperative period. Objective: To describe the incidence of the SAS in postoperative patients with activation of code 77. Material and methods: Observational, transversal, retrospective and descriptive study, included patients with indication of activation of code 77 during their immediate postoperative time, and transferred to the Intensive Care Unit, during the period from January to December 2015. Results: 68\% of the sample obtained a SAS greater than or equal to 7 points (stable patient), and 32\% a SAS less than or equal to 6 (unstable). The incidence of SAS less than or equal to 6 points for the period of the study was about 12 cases per 100 persons intervened with a sensibility of $68 \%$. Conclusions: SAS should be taken as another criteria for the activation of code 77 in those patients with a result lower than 6 points; during the immediate postoperative period, allowing a timely management of postoperative complications and a reduction on the risk of morbidity and mortality.

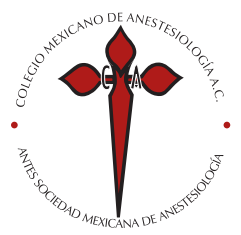

Palabras clave:

Apgar quirúrgico, cuidados postanestésicos, complicaciones postquirúrgicas, código 77.

Keywords:

Apgar score, postsurgical care, postoperative complications, code 77.

\footnotetext{
* Médico Residente de Anestesiología del Hospital Ángeles Pedregal. Facultad Mexicana de Medicina, Universidad La Salle México. México. ‡ Médico del Departamento de Anestesiología del Hospital Ángeles Pedregal. México.

$\S$ Médico del Departamento de Ginecología y Obstetricia del Hospital Ángeles Pedregal. México. " Médico Especialista en Medicina del Enfermo Adulto en Estado Crítico del Hospital Star Médica Querétaro. México. ॥ Profesor Titular del Curso de Anestesiología del Hospital Ángeles Pedregal. México.

Correspondencia:

Dra. Erika Ramírez-Patiño Hospital Ángeles Pedregal, Departamento de Enseñanza. Camino a Sta. Teresa Núm. 1055-S, Héroes de Padierna, 10700, Ciudad de México, México. E-mail: erika.rpat@gmail.com
}

Recibido: 27-10-2021

Aceptado: 10-11-2021

\section{INTRODUCCIÓN}

L a clasificación de Apgar comenzó a practicarse desde el año 1953 en la rama de anestesiología y neonatología, cuando la doctora Virginia Apgar propuso un método clínico para evaluar el estado físico de los neonatos al minuto y a los cinco minutos después del nacimiento ${ }^{(1)}$. Dicho método describe la condición inmediata del recién nacido y su aplicación a los cinco minutos del postnacimiento y se considera como el mejor predictor de supervivencia infantil ${ }^{(2,3)}$.

La atención quirúrgica es un componente vital de la atención médica universal, sin embargo, no existe una medida clínicamente significativa de los resultados quirúrgicos que se pueda aplicar a nivel mundial ${ }^{(4)}$. Es así como nace en cirugía la necesidad de tener un medidor 
de rutina confiable y fácil de aplicar, que permita conocer las condiciones generales del paciente inmediatamente después de un procedimiento quirúrgico. El Apgar quirúrgico (AQ), conocido como SAS (por sus siglas en inglés Surgical Apgar Score), es un sistema de 10 puntos que involucra tres variables intraoperatorias: menor frecuencia cardíaca, menor presión arterial media y pérdida de sangre $^{(5)}$, que pueden predecir de manera efectiva complicaciones mayores o incluso la posibilidad de muerte postoperatoria en el período postoperatorio inmediato y hasta dentro de los primeros 30 días después de realizada la cirugía $^{(6)}$. El puntaje obtenido es inversamente proporcional al riesgo potencial de complicaciones, es decir, a menor puntaje de AQ existe una mayor incidencia de complicaciones y a mayor puntaje obtenido, menor probabilidad de las mismas. Diversos estudios sugieren que debe utilizarse para guiar objetivamente los cuidados postoperatorios $^{(7,8)}$; sin embargo, a través de la literatura

Tabla 1: Criterios para la activación del código 77 (equipo de respuesta rápida) en el Hospital Ángeles Pedregal.

$\begin{aligned} & \text { 1. Impresión de la gravedad } \\ & \text { 2. } \text { Dificultad respiratoria } \\ & \text { 3. } \text { Saturación de oxígeno }<92 \% \text { con oxígeno suplementario por } \\ & \text { puntas nasales } \\ & \text { 4. } \text { Frecuencia respiratoria }(<120>30 \mathrm{rpm}) \\ & 5 . \text { Frecuencia cardíaca }(<400130 \mathrm{lpm}) \\ & 6 . \text { Tensión arterial sistólica }(<90 \mathrm{a}>180 \mathrm{mmHg}) \\ & 7 . \text { Gasto urinario }(<500>200 \mathrm{~mL} / \mathrm{h}) \\ & \text { 8. } \text { Cambio agudo del estado de alerta (confusión o desorientación, } \\ & \text { 9. } \text { estupor y coma, crisis convulsivas, focalización) } \\ & \text { 10. } \text { Dolor torácico }\end{aligned}$

$\mathrm{rpm}=$ respiraciones por minuto; $\mathrm{lpm}=$ latidos por minuto; $\mathrm{mmHg}=$ milímetros de mercurio; $\mathrm{mL} / \mathrm{h}=$ mililitros por hora.

\begin{tabular}{|lc}
\multicolumn{2}{c}{$\begin{array}{c}\text { Tabla 2: Distribución de casos postoperatorios de } \\
\text { acuerdo con la especialidad quirúrgica. }\end{array}$} \\
\hline Especialidad quirúrgica & Número de casos postoperatorios \\
\hline Cirugía General & 23 \\
Cirugía Plástica & 2 \\
Cirugía de Tórax & 1 \\
Gastrocirugía & 2 \\
Ginecología Quirúrgica & 5 \\
Nefrología & 1 \\
Neurocirugía & 1 \\
Oncología Quirúrgica & 5 \\
Ortopedia & 11 \\
Otorrinolaringología & 2 \\
Urología & 5
\end{tabular}

\section{Tabla 3: Criterios de inclusión y exclusión.}

\section{Criterios de inclusión}

Pacientes de entre 18 y 100 años de edad de ambos sexos Pacientes postoperados con activación de código 77 con 0 sin evaluación de Apgar quirúrgico en el postoperatorio inmediato Pacientes postoperados con activación de código 77 trasladados a Unidad de Terapia Intensiva

Criterios de exclusión

Pacientes menores de 18 o mayores a 100 años de edad Pacientes postoperados sin activación del código 77 Pacientes postoperados con activación del código 77 sin traslado a la Unidad de Terapia Intensiva Pacientes con activación del código 77 sin antecedente quirúrgico inmediato

respecto a esta escala no se ha determinado un valor predictivo positivo o negativo durante el transcurso del período de 30 días.

Dentro de los estándares de acreditación de hospitales del Joint Commission International (JCI, al día de hoy la organización con más experiencia en acreditación sanitaria de todo el mundo) se encuentra mejorar la calidad de atención y seguridad al paciente ${ }^{(9)}$. Es por ello que a partir del año 2014 se implementó en el Hospital Ángeles Pedregal (HAP) el concepto de equipo de respuesta rápida (ERR) con el voceo del código 77, apegándose a los estándares de Acceso y Continuidad de la Atención (ACC) 2.3 de la JCI en donde la admisión a servicios de cuidados intensivos es determinada por criterios establecidos. El ERR lleva a cabo un plan de acción inmediato a través de la identificación de datos de deterioro clínico en el paciente hospitalizado (Tabla 1), valorando y determinando su ingreso a la Unidad de Terapia Intensiva (UTI). La implementación de dicho plan consiguió buenos resultados desde su primer año de instauración, aumentando la supervivencia de los pacientes admitidos en la UTI hasta en $37.7 \%$ comparados con los que no fueron valorados por el $\mathrm{ERR}^{(10)}$.

El objetivo del estudio es describir la incidencia del puntaje de AQ en los pacientes postoperados con activación de código 77 que requirieron manejo en la UTI del HAP y analizar si el puntaje de AQ del paciente debe considerarse un criterio más para la activación del código 77 .

\section{MATERIAL Y MÉTODOS}

Se realizó un estudio observacional, descriptivo, retrospectivo de corte que incluyó a los pacientes con indicación de activación del código 77 en su postoperatorio inmediato de diversas especialidades y subespecialidades que requirieron traslado a la Unidad de Cuidados Intensivos (Tabla 2) du- 
rante el período de enero a diciembre de 2015 en el Hospital Ángeles Pedregal. Los criterios de inclusión y exclusión se resumen en la Tabla 3.

Se revisó la información obtenida de 147 expedientes de pacientes con ingreso a la Unidad de Terapia Intensiva y activación del código 77, y se incluyó el 39\% (58) (Figura 1). Las variables analizadas se obtuvieron de la revisión de las hojas de registro transoperatorio, para el posterior cálculo del AQ a través de los siguientes tres parámetros: frecuencia cardíaca más baja, presión arterial media más baja y sangrado transoperatorio (Tabla 4); se consideró un puntaje de $\mathrm{AQ} \geq$ 7 puntos como paciente estable y un puntaje $\leq 6$ como paciente inestable; y de los expedientes clínicos se extrajeron los datos para las complicaciones según la especialidad y subespecialidad.

El análisis estadístico se realizó mediante la incorporación de los datos en una matriz creada en Microsoft Excel y posterior importación de éstos al software estadístico SPSS de IBM versión 23, con el cual se generaron estadísticas descriptivas (frecuencias y proporciones) para las variables cualitativas.

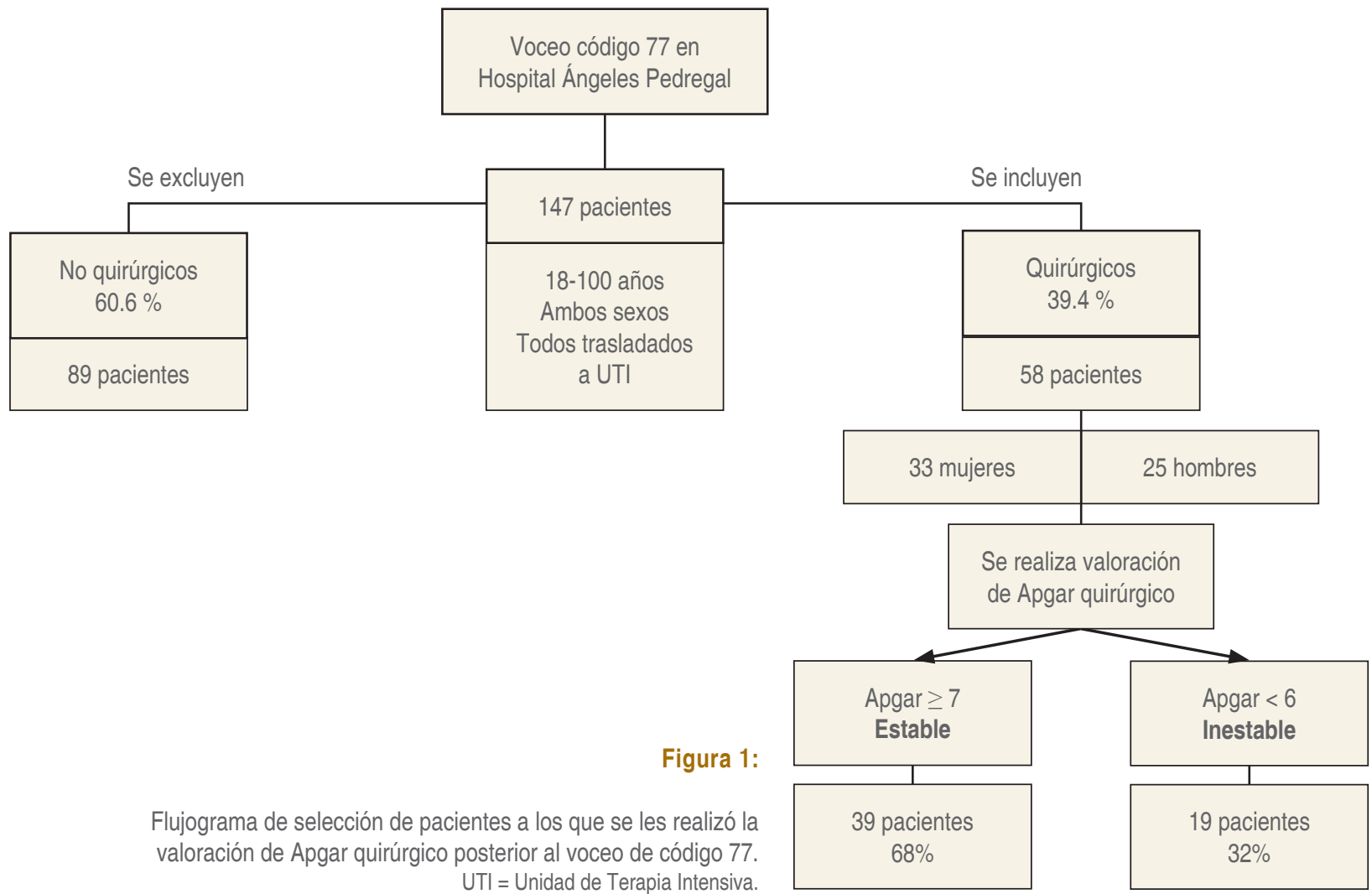

Tabla 4: Escala de Apgar quirúrgico.

Puntos

\begin{tabular}{|c|c|c|c|c|c|}
\hline & \\
\hline & 0 & 1 & 2 & 3 & 4 \\
\hline Pérdida sanguínea $(\mathrm{mL})$ & $>1,000$ & $601-1,000$ & $101-600$ & $<100$ & \\
\hline PAM más baja (mmHg) & $<40$ & $40-54$ & $55-69$ & $>70$ & \\
\hline \multirow[t]{2}{*}{ FC más baja (Ipm) } & $>85$ & $76-85$ & $66-75$ & $56-65$ & $<55$ \\
\hline & \multicolumn{5}{|c|}{ Puntuación máxima = 10 puntos } \\
\hline
\end{tabular}

PAM = presión arterial media; FC = frecuencia cardíaca; $\mathrm{mmHg}=$ milímetros de mercurio; 1 pm = latidos por minuto. 


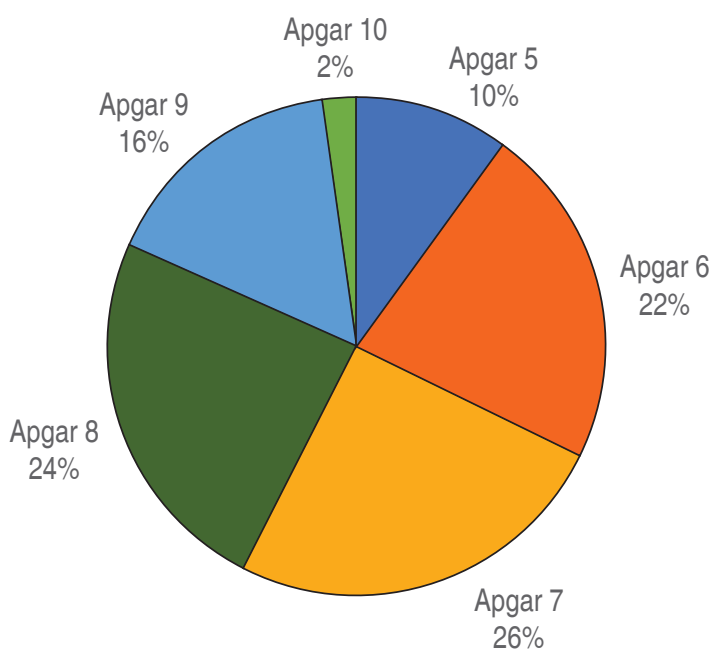

Figura 2: Puntaje de Apgar quirúrgico.

Tabla 5: Distribución del puntaje de Apgar quirúrgico obtenido.

\begin{tabular}{lccccc} 
& \multicolumn{5}{c}{ Puntos } \\
\cline { 2 - 6 } & 0 & 1 & 2 & 3 & 4 \\
\hline Pérdida sanguínea & 0 & 0 & 40 & 18 & \\
PAM más baja & 0 & 7 & 24 & 27 & \\
FC más baja & 7 & 7 & 13 & 13 & 18 \\
\hline
\end{tabular}

PAM = presión arterial media; FC = frecuencia cardíaca.

\section{RESULTADOS}

Se contó con 58 pacientes de los cuales 68\% (39) obtuvieron un Apgar quirúrgico mayor o igual a 7 puntos (estables) y $32 \%$ (19) un Apgar quirúrgico menor o igual a 6 (inestables) con activación del código 77 y posterior traslado a terapia intensiva (Figura 2 y Tabla 5). La incidencia del Apgar quirúrgico 6 para el período de estudio fue de 12 casos por cada 100 personas intervenidas con una sensibilidad del $68 \%$.

En cuanto a la prevalencia de complicaciones, según la especialidad quirúrgica, fue de $39.6 \%$ para Cirugía General y $18.9 \%$ para Traumatología y ortopedia; Ginecología, Urología y Oncocirugía tuvieron 8.6\% cada una (Figura 3).

Las complicaciones postquirúrgicas más frecuentes fueron choque (hemorrágico, séptico y neurogénico) y complicaciones cardiovasculares (infarto agudo al miocardio, insuficiencia cardíaca, arritmias, angina inestable y tromboembolia pulmonar) (Figura 4).

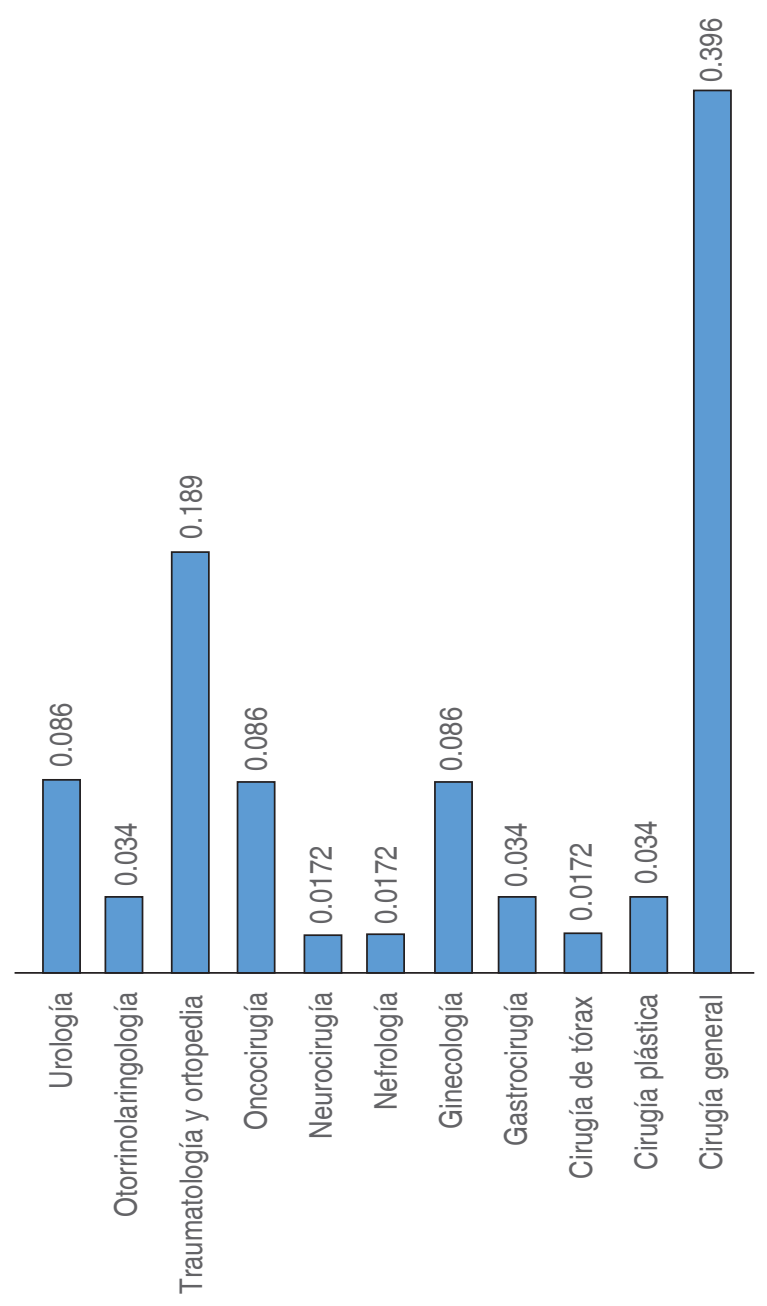

Figura 3: Porcentaje de prevalencia de complicaciones de acuerdo con la especialidad quirúrgica.

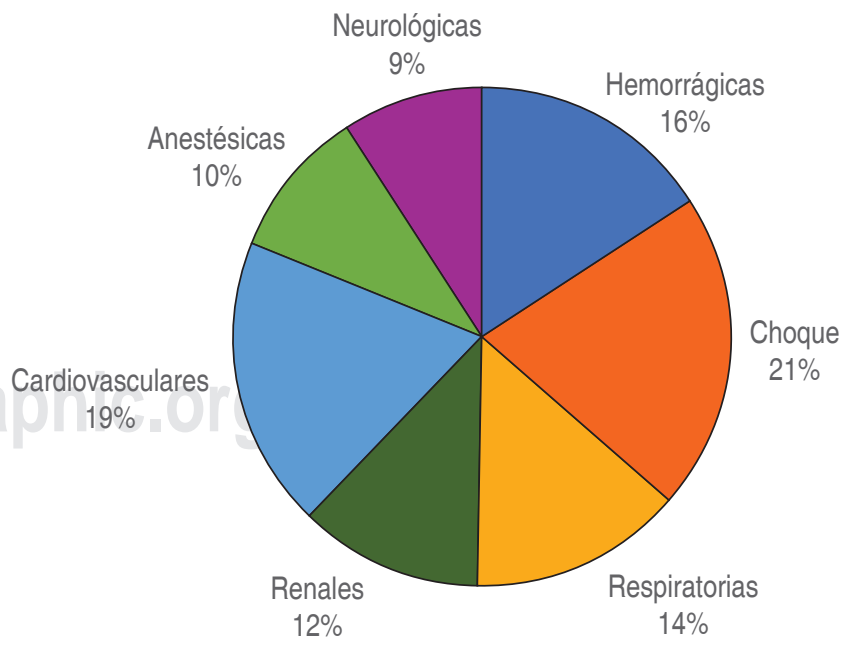

Figura 4: Complicaciones postquirúrgicas más frecuentes. 


\section{DISCUSIÓN}

El 100\% de los casos estudiados se trasladó a la Unidad de Terapia Intensiva posterior a la activación del código 77, de los cuales ninguno de los casos se activó tomando el AQ como un parámetro para su activación. En $100 \%$ de los casos, la activación del código 77 fue desde su estancia en hospitalización.

Un puntaje menor a 6 puntos se considera un paciente inestable, lo que significa que tiene alta probabilidad de presentar complicaciones inmediatas, por lo que consideramos que debe evaluarse su ingreso directo a la Unidad de Terapia Intermedia para su seguimiento inmediato, en lugar de su ingreso a hospitalización.
Una puntuación mayor o igual a 7 se considera de menor riesgo de resultados adversos postoperatorios, por tanto deben evaluarse los otros criterios para la activación del código 77.

El Apgar quirúrgico debe realizarse en todo paciente durante los primeros 30 minutos postoperatorios en la sala de cuidados postanestésicos; se propone que se establezca que un puntaje AQ igual o menor a 6 puntos signifique activación del código 77, y por lo tanto que el paciente debiera continuar su seguimiento y manejo por lo menos en la Unidad de Cuidados Intermedios, con el fin de proporcionar un manejo oportuno e inmediato de las probables complicaciones que puedan presentarse, disminuyendo con esto el riesgo de morbilidad y/o mortalidad ${ }^{(11)}$

\section{REFERENCIAS}

1. Casey BM, McIntire DD, Leveno KJ. The continuing value of the Apgar score for the assessment of newborn infants. N Eng J Med. 2001;344:467-471.

2. Papile LA. The Apgar score in the $21^{\text {st }}$ century. N Eng J Med. 2001;344:519-520.

3. Gawande AA, Kwaan MR, Regenbogen SE, Lipsitz SA, Zinner MJ. An Apgar score for surgery. J Am Coll Surg. 2004;4:201-206.

4. Regenbogen SE, Lancaster RT, Lipsitz SR, Greenberg CC, Hutter MM, Gawande AA. Does the Surgical Apgar Score measure intraoperative performance? Ann Surg. 2008;248:320-328.

5. Padilla Leal KE, Flores Guerrero JE, Medina Franco H. Apgar quirúrgico como predictor de complicaciones en cirugía oncológica gastrointestinal. Rev Gastroenterol Mex. 2021;86:259-264. doi: 10.1016/j.rgmx.2020.06.004

6. Reynolds PQ, Sanders NW, Schildcrout JS, Mercaldo ND, St Jacques PJ. Expansion of the surgical Apgar score across all surgical subspecialties as a means to predict postoperative mortality. Anesthesiology. 2011;114:1305-1312.
7. Haynes AB, Regenbogen SE, Weiser TG, Lipsitz SR, Dziekan G, Berry WR, et al. Surgical outcome measurement for a global patient population: validation of the Surgical Apgar Score in 8 countries. Surgery. 2011;149:519-524.

8. Haddow JB, Adwan H, Clark SE, Tayeh S, Antonowicz SS, Jayia P, et al. Use of the surgical Apgar score to guide postoperative care. Ann R Coll Surg Engl. 2014;96:352-358.

9. Joint Commission International. Estándares de acreditación para hospitales de Joint Commission International. 5a edición. Joint Commission International; 2014. Disponible en: http://www.jcrinc.com

10. Elguea Echavarría PA, Hernández Cortés C, Ramírez Ramírez L, García Gómez N, López López C, Gómez Gómez B, et al. Efectividad del equipo de respuesta rápida en el Hospital Ángeles Pedregal. Acta Méd Grupo Ángeles. 2017;15:181-188.

11. Castro MM, Espitia E, Domínguez LC. Evaluación del Apgar quirúrgico en la predicción de complicaciones y muerte perioperatoria: análisis prospectivo en un centro de cuarto nivel de Bogotá. Rev Colomb Cir. 2014;29:213-221. 